\title{
A methodology for managing integration problems with CIMOSA
}

\author{
A.J.R. Zwegers, Eindhoven University of Technology \\ T.A.G. Gransier, TNO-TPD and Eindhoven University of Technology \\ PO Box 513, $5600 \mathrm{MB}$ Eindhoven, The Netherlands \\ Tel. +31 40 474370,Fax+3140436492,Emailazw@bdk.tue.nl
}

\begin{abstract}
The objective of this paper is to present a methodology for re-engineering processes in which the architectural framework CIMOSA supports an organisation in managing its integration difficulties. New market requirements demand new production strategies. Therefore, enterprises follow re-engineering processes and are faced with consequent integration problems. We investigated the re-engineering processes in three European enterprises that have applied CIMOSA in changing their manufacturing systems. Important factors in these re-engineering processes are the CIMOSA framework, the logical architecture, and the physical architecture. Appropriate usage of the CIMOSA modelling framework and the integrating infrastructure contributes to business integration and application integration. Although the CIMOSA framework has some gaps, the concepts behind it and the experiences gained show that it enables companies to manage integration difficulties.
\end{abstract}

\section{Keywords}

Enterprise integration, CIMOSA, Computer Integrating Manufacturing, Industrial automation

\section{INTRODUCTION}

Enterprises face integration problems due to necessary internal adaptations to cope with severe competition in the global marketplace. In order to survive at the global market, efficient operation and innovative management of change are essential. Heterogeneous manufacturing and information systems are of serious concern in improving the current enterprise operation. In addition, since companies try to meet competition and to exploit new opportunities, enterprise operations have to evolve continuously. Evolving enterprise operations create new heterogeneities of legacy and new technology systems, so that integration problems are amplified.

The objective of this paper is to present a methodology for re-engineering processes in which the architectural framework CIMOSA - Computer Integrated Manufacturing - Open System Architecture - supports an organisation in managing integration problems. CIMOSA 
claims to solve the above-mentioned problems (AMICE, 1993); it aims to offer support on two kinds of integration, namely 'business integration' and 'application integration'. Business integration is concerned with the coordination of operational and control processes in order to satisfy business objectives. Application integration, which affects the control of applications, means that cooperation between humans, machines and software programs has to be established. This paper shows how the use of CIMOSA supports an organisation in managing its integration difficulties.

Research was conducted in three industrial organisations throughout Europe, namely in the Greek aluminium foundry Elval, in the French car plant Renault, and in the German machine tool factory Traub. Results were obtained by document analyses and interviews with people from the three companies that applied CIMOSA from 1991 to 1994 in the context of the VOICE projects (Esprit project numbers 5510 and 6682). Detailed information about Elval, Renault and Traub is given by Arabatzis, Piérard and Schlotz respectively (VOICE, 1995).

This paper is organised as follows. In the next section, we present the concepts that play a role in an organisation's approach to the migration path from a current Computer Integrated Manufacturing (CIM) system to a future system. These concepts, namely a CIM system's logical and physical architecture, and CIMOSA, serve as a framework for the description of the system engineering activities. After this, a methodology for system engineering supported by the architectural framework CIMOSA is presented. Furthermore, we show how to accomplish enterprise integration during system engineering by means of CIMOSA. We conclude this paper with a discussion on our findings.

\section{ENGINEERING APPROACH}

When necessary, enterprises decide to change their manufacturing systems. Modifications to current CIM systems are required because of changing market requirements, business objectives, business requirements, manufacturing concepts or available technology. The decision to manufacture new products, might for example result in changes in the existing CIM system. The manufacturing systems that are in operation today will be re-engineered at some point in time to turn into next-generation manufacturing systems, so that current integrated systems become future heritage systems.

We look at a manufacturing system from a logical and physical point of view, i.e. we distinguish logical components and physical components. During the re-engineering process these components and their interfaces are defined. In other words, the re-engineering process involves the design of a logical and a physical architecture.

Elval, Renault and Traub applied the CIMOSA architectural framework in their re-engineering processes. Figure 1 visualises the approach of the three companies. They defined logical architectures by means of the CIMOSA modelling framework. The physical systems that provide enterprise operation according to the defined models, were designed with the help of the CIMOSA integrating infrastructure. Finally, the CIM systems were implemented and transferred into operation.

In the remainder of this section, the three concepts, namely the CIMOSA architectural framework, logical architectures and physical architectures, are presented in more detail. In the next section, we illustrate the roles of these concepts during a re-engineering process. 


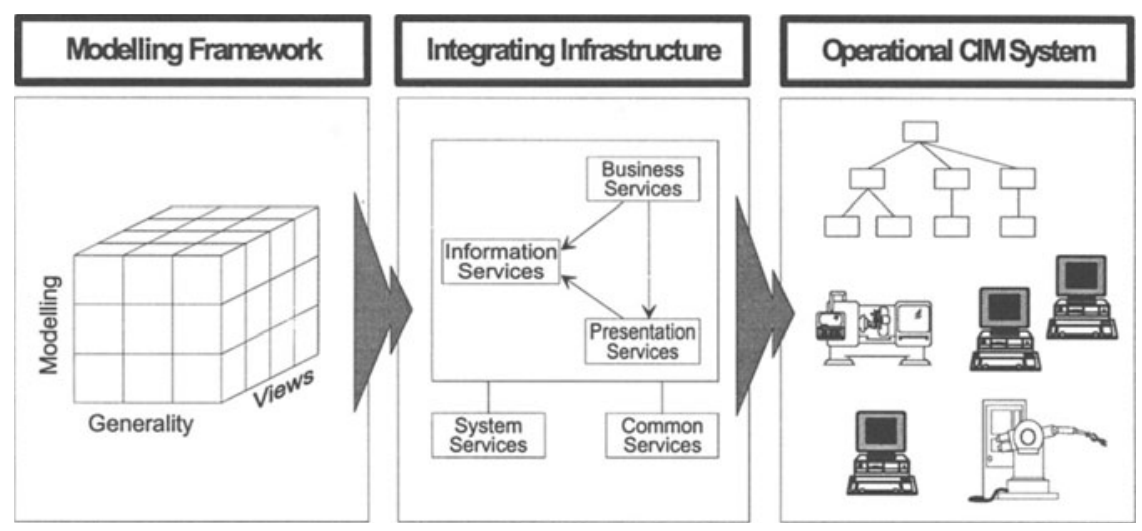

Figure 1 Engineering approach.

\subsection{CIMOSA}

The first concept is the CIMOSA architectural framework, which consists of the CIMOSA modelling framework and the integrating infrastructure.

\section{CIMOSA modelling framework}

In Figure 1, the modelling framework is represented by the CIMOSA cube. The cube allows to model different aspects and views of an enterprise. This three-dimensional framework has a dimension of generality, a dimension of enterprise models, and a dimension of views:

- The generality dimension is concerned with the degree of particularisation. It goes from generic building blocks to their aggregation into a model of a specific enterprise domain.

- The modelling dimension provides the modelling support for the system life cycle, starting from statements of requirements to a description of the system implementation.

- The view dimension offers the possibility to work with sub-models representing different aspects of the enterprise.

\section{CIMOSA integrating infrastructure}

The CIMOSA integrating infrastructure enables CIMOSA models to be executed; it allows the control and monitoring of enterprise operations as described in the models. Furthermore, it provides a unifying software platform to achieve integration of heterogeneous hardware and software components of the CIM system.

The integrating infrastructure is made of a number of system-wide, generic services. The business services control the enterprise operations according to the model. The information services provide for data access, data integration and data manipulation, and the presentation services act as a standardised interface to humans, machines, and software applications. A product that is connected to the presentation services, can be attached and removed without changing any other part of the information technology environment. Other services are the common services and the system management services. In Figure 1 the integrating infrastructure and the mentioned services are shown. 


\subsection{Logical architecture}

The purpose of an architecture is to structure a complex system. It structures a system into modules and it determines the modules' interfaces. The logical architecture defines the functions of system components and their interfaces. Functions are assigned to components, allowed inputs and outputs are defined, and the relations between components are specified.

The importance of a logical architecture is derived from its relationship with the manufacturing system. The architecture determines how functions are distributed and coordinated. By fixing the functionality of the CIM system, a logical architecture determines the effectiveness of the system. However, changing market requirements make new, higher demands upon enterprises, which have to adjust their manufacturing systems according to the new objectives. Besides determining the system's effectiveness, the logical architecture is perhaps even more important because it establishes the limitations or possibilities for changing the system's functionality and effectiveness in the future (Dilts, 1991).

\subsection{Physical architecture}

The third concept is the physical architecture of a manufacturing system. Whereas the logical architecture defines component functions and their interfaces, the physical architecture defines physical components and their interactions. Examples of physical components are concrete objects such as computers, programmable logic controllers, networks, and manufacturing resources, but also nontangible objects such as software and NC-programs.

The design of a physical architecture is influenced by the evolving technology. On one hand, the technology opens opportunities for system development; on the other hand, system architectures have to be able to take the future opportunities of evolving technology into account. Future information and manufacturing technology components have to be integrated into the current system, since new objectives might result in the application of new technology. Both the logical and the physical architecture must provide flexibility to the system, so that the system can be adjusted to the new technology components.

\section{SYSTEM ENGINEERING}

In this section, a methodology for re-engineering processes is presented, based on the experiences gained by the three industrial organisations Elval, Renault and Traub during their re-engineering processes. For a more extended description the reader is referred to Zwegers (VOICE, 1995). In the next section, we focus on enterprise integration during system engineering.

The phases of the re-engineering process are schematically shown in Figure 2 and are described in more detail in the following subsections.

\subsection{Determination of objectives}

Enterprises change their production systems driven by certain objectives. At a specific moment, an organisation is dissatisfied with its current manufacturing system. Then, new 
objectives are determined for (part of) the system. These objectives are derived from the company's business strategy and are the starting points for system development.

\subsection{Definition of requirements}

In the previous phase, the decision is taken to change the current CIM system. This phase is concerned with the definition of requirements for the future system.

Requirements are defined in a logical sense for the application to be developed, and in a technical sense for the application's underlying infrastructure. In other words, requirements are defined for both the logical and physical components of the manufacturing system. For the logical components, requirements are described in terms of a scenario. In this scenario, the needed functions and

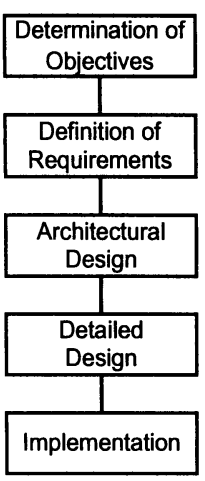

Figure 2 System engineering phases. information flows, and their place in the total manufacturing system are outlined. For the physical components, technical requirements are given. Besides the users' current needs, requirements take into consideration the existing manufacturing system, strategic aspects like standards, the factory environment and production process constraints.

\subsection{Architectural design}

After requirements are defined, the design of the CIM system commences. We have distributed the design activities over two phases: architectural design and detailed design. In the architectural design phase, the system's logical and physical architecture are defined, supported by the CIMOSA framework. In the detailed design phase, the system is worked out in more detail, based on the specified architectures.

Before the logical and physical architecture of the future system are specified, the existing manufacturing system is analysed. Knowledge acquisition can be carried out by modelling the current manufacturing system using the CIMOSA modelling framework. The requirements definition level and, if more detailed information is required, the design specification level allow to obtain the necessary information for the analysis of the current CIM system.

The CIMOSA modelling framework supports a user in defining his CIM system's logical architecture. The (particular) requirements definition level of the modelling framework allows the user to model the functional scenario description, that was defined previously. By modelling, the user structures the system component functions, defines the components' allowed inputs and outputs, and specifies the relations between these components. In other words, by modelling at requirements definition level a logical architecture is designed.

Along with defining a logical architecture, the designer also outlines a physical architecture, whether or not influenced by the existing infrastructure. When defining logical components, one immediately maps these components on physical ones; the logical architecture is depicted on the physical architecture. For instance, when the designer defines a control function, he might decide to execute this function on a workstation. In addition, the designer determines the interaction of this component with other physical components. 


\subsection{Detailed design}

In the second design phase, the CIM system is specified in more detail, elaborating on the architectures that were defined in the architectural design phase. Both architecture specifications are further decomposed and worked out into detailed system designs.

By means of the CIMOSA design specification modelling level, a designer is able to detail a manufacturing system's functionality, taking the model at requirements level as starting point. The requirements definition level supports a user in the definition of his CIM system's logical architecture, whereas the role of the design level is to restructure, detail and optimise the required functionality in a consistent model. System optimisation can be supported by simulation, taking all business and technical constraints into account. By the specification of a model at design level, a designer describes the full functionality of a CIM system, while staying within the specification of the logical architecture. If the model at design specification level reveals inconsistencies, or the model lacks optimisation, it might be necessary to adjust the model at requirements definition level.

In addition to detailing the functionality of the system, the designer specifies the technology to be employed in order to achieve the required system functionality. Simply stated, CIMOSA prescribes that for each of the most detailed specified functions, called 'functional operations', a resource is assigned that provides the required capabilities. The prime task of the design specification modelling level is to establish a set of (logical) resources that together provide the total set of required capabilities. Some of these required capabilities are offered by the generic services of the integrating infrastructure.

A refinement of specified functions is accompanied by a refinement of the physical resources that provide the desired functions. An enterprise looks for adequate products, either commercial ones or user developed. The physical components, which are defined in the architectural design phase, are specified completely. For this specification, the definitions of the CIMOSA integrating infrastructure are used. Then, the products that fulfil the design specifications are selected, considering enterprise policies and constraints. CIMOSA states that the final build/buy decisions should result in a model that describes the implemented, physical system. Appropriately, CIMOSA calls this model the implementation description model. However, since this part of the modelling framework has been defined recently, we do not have any experience with it.

\subsection{Implementation}

The final phase constitutes of the implementation and release for operation of the specified CIM system. Implementation is based upon the results and decisions of the previous phases.

Implementation activities concern those tasks needed to bring the system into operation. During the implementation phase, the necessary new physical components are procured or built. These components are tested on a testbed and the correctness of the underlying logical model is verified. When the system passes the tests with satisfactory results, the system is prepared for transfer to production. Essential activities such as operator training and acceptance testing are carried out before transfer to production. Finally, the accepted CIM system is released for operation, after which it manufactures products until it is replaced by the next CIM system. 


\section{ENTERPRISE INTEGRATION}

In the previous section, a methodology for system engineering is given, in which the role of the CIMOSA architectural framework is illustrated. Based on the experiences gained, we indicate in this section how enterprise integration can be achieved by means of CIMOSA. We proceed with the distinction in enterprise integration as presented in the introduction, particularly business integration and application integration.

\subsection{Business integration}

The CIMOSA models allow the user to design logical architectures. During the architectural design phase, the CIMOSA user is able to acquire knowledge of his current CIM system by means of modelling at the requirements definition level. This knowledge is needed in order to analyse the existing system. Then, the model is changed to take required modifications into account. By means of the specification of components that represent operational and control functions, the inputs and outputs of components, and interfaces between components, a logical architecture is defined.

The definition of a sound logical architecture, upon which the further design of the system is based, is the key to business integration. CIMOSA takes a modular approach to integration, i.e. enterprise operation is modelled as a set of cooperating processes which exchange results and requests. Only this exchanged data needs to have a representation that is common between the cooperating processes. In addition, CIMOSA provides the means to manage processes that are inherited from the past. By employing models for identification, analysis and coordination of existing and new functions, required logical architectures can be designed that define modular components and their interfaces. When the design and implementation of the CIM system follows the architecture specification, the requested integration of business processes will be obtained. In this way, the required level of business integration is achieved, which is reflected by the coordination of operational and control processes that fulfil the business objectives.

\subsection{Application integration}

The other aspect of enterprise integration, application integration, is concerned with the usage of information technology to provide interoperation between manufacturing resources. Whereas business integration can be regarded as the integration of logical components, application integration affects the integration of physical components.

Application integration is supported by the CIMOSA integrating infrastructure. Cooperation between humans, software programs, and machines has to be established. The integrating infrastructure provides services to integrate the enterprise's heterogeneous manufacturing and information systems in order to satisfy the business needs as identified with the help of the modelling framework. The CIMOSA specification of the integrating infrastructure can be seen as a reference model. Physical components that are designed and implemented according to this reference model provide desired features such as interoperability, portability, connectivity, and transparency. 


\section{DISCUSSION}

Although the engineering process is presented as a sequence of phases, this does not imply that it follows a waterfall model. In contrary, designing CIM systems is an iterative process, with frequent jumps between the phases. Iterations are necessary because of increased insight, unexpected events, etc. Therefore, the presented methodology should be considered as a presentation of advisable activities, and not as a prescription of mandatory phases.

In addition, jumps between the logical (or functional) domain and the physical domain occur. The specification of both logical and physical components during an engineering process go hand in hand. A refinement of logical components is accompanied by a refinement of the physical components. Figure 3 presents design as the refinement process that interlinks the logical and the physical domain. Note that this observation is confirmed by Suh's theory on designing products (Suh, 1990).

Although the CIMOSA framework has some gaps, it enables companies to manage integration difficulties. The CIMOSA modelling framework is not complete; for instance, the three companies did not have the definition of the implementation description modelling level at their disposal. Furthermore, the integrating infrastructure was not specified completely. However, CIMOSA suffers most from the lack of commercial products that fulfil the CIMOSA (integrating infrastructure) specifications. Nevertheless, the concepts behind it and the positive experiences gained by the three industrial organisations show that CIMOSA provides valuable contributions to managing integration problems.

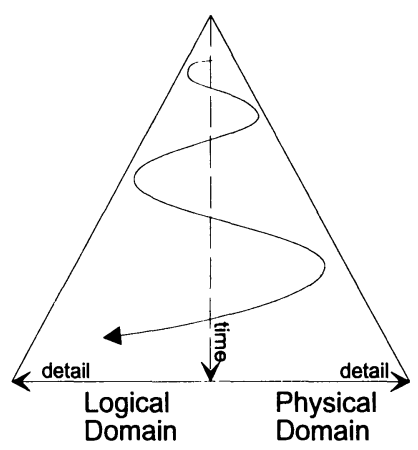

Figure 3 The design process.

\section{REFERENCES}

AMICE Consortium (1993) CIMOSA: Open System Architecture for CIM. Springer-Verlag, Berlin.

Dilts, D.M., Boyd, N.P. and Whorms, H.H. (1991) The evolution of control architectures for automated manufacturing systems. Journal of Manufacturing Systems, vol. 10, no. 1, 79-93. Suh, N.P. (1990) The principles of design. Oxford University Press, New York.

VOICE Consortium (1995), Special issue on application and validation of CIMOSA. Computers in Industry, to be published.

\section{BIOGRAPHY}

Arian Zwegers has been working on the Esprit project VOICE II as a Ph.D. student from the Eindhoven University of Technology. His research interests include architectures in general, and control architectures in particular.

Theo Gransier has been working on both VOICE projects. First, he was responsible for the technical coordination of the modelling part. From 1993, he was project manager of the VOICE II project. His main research interests are distributed control architectures. 